\title{
Classification Scheme Item
}

National Cancer Institute

\section{Source}

National Cancer Institute. Classification Scheme Item. NCI Thesaurus. Code C41080.

A component of content in a Classification Scheme. This may be a node in a taxonomy or ontology or a term in a thesaurus, etc. 